\title{
Magnetic resonance imaging $\mathrm{MRI}$ of the brain spares a patient with temporal lobe dual pathology from invasive electroencephalography IEEG prior to epilepsy surgery
}

\begin{abstract}
Focal cortical dysplasia FCD can be detected in the temporal pole TP of patients with temporal lobe epilepsy TLE, The association of TP FCD and hippocampal sclerosis HS is increasingly recognized especially in young patients. High resolution magnetic resonance imaging MRI of the brain can identify macroscopic TP FCD in the preoperative evaluation for epilepsy surgery. Favorable surgical outcome is achieved when both lesions of the dual pathology are removed .In this case report we describe a twelve years old boy with pharmaco-resistant TLE. An initial MRI of the brain showed right HS .The clinical and neurophysiological features were atypical for HS. High resolution Three Tesla MRI brain performed prior to invasive electroencephalography IEEG and identified right TP FCD in additional right HS. The patient underwent directly epilepsy surgery with intraoperative electrocorticography and right temporal lobectomy with resection of the right TP is performed. The histopathology confirmed the right HS and FCD type III a. The patient had no postoperative complications and became seizure free. He remained seizure free on small dose of Lamotrigine after five years of follow up after epilepsy surgery.
\end{abstract}

Keywords: focal cortical dysplasia, pharmaco-resistant, magnetic resonance imaging, patients, monotherapy
Volume 8 Issue 4 - 2018

\author{
Adulelah Nasser AL Jasser, ' Sonia Abdul- \\ Mannan Khan ${ }^{2}$ \\ 'King Saud University, Saudi Arabia \\ ${ }^{2}$ Consultant Neurologist/Epileptologist, Department of \\ Neurology, Saudi Arabia
}

\begin{abstract}
Correspondence: Sonia AS Khan, Consultant Neurologist/ Epileptologist, Head of the Epilepsy Division, Neurology Department, PSMMC, Riyadh, Saudi Arabia, Tel 009665054466 I0, Emailskrmasa5@gmail.com
\end{abstract}

Received: March 26, 2018 | Published: July 05, 2018
Abbreviations: FCD, focal cortical dysplasia; MRI, magnetic resonance imaging; TP, temporal pole; IEEG, invasive electroencephalography; EMU, epilepsy monitoring unit; TLE, temporal lobe epilepsy; AMTLR, anterior mesial temporal lobe resection; $\mathrm{MCD}$, malformations of cortical development

\section{Introduction}

Temporal lobe epilepsy with hippocampal sclerosis HS is a distinct clinical syndrome with classical clinical, neurophysiological and neuroimaging features. ${ }^{1}$ Focal cortical dysplasia FCD in the extrahippocampal regions is common with HS especially in younger patients and temporal polar cortex TP is frequently involved in seizure onset. $^{2}$ Patients with such dual pathology may achieve favorable outcome from epilepsy surgery provided that both pathologies are removed. ${ }^{2}$ The objective of this case report is to demonstrate the association of $\mathrm{HS}$ and FCD, emphasize the role of high resolution magnetic resonance imaging of the brain in the diagnosis of dual pathology with temporal pole FCD, distinguish preoperatively the clinical and neurophysiological features of this association and the impact on seizure control post epilepsy surgery .

\section{Case presentation}

A twelve years old right handed boy a school student referred to the epilepsy monitoring unit for epilepsy surgery evaluation of pharmacoresistant epilepsy that failed several antiepileptic drugs monotherapy and poly therapy trials. The patient is a product of full term normal spontaneous vaginal delivery with normal mile stones. He had febrile convulsions at the age of one year with no recurrence and had family history of epilepsy with one sibling and one paternal cousin had epilepsy with generalized tonic clonic seizures in remission on anti epileptic drugs. The first generalized tonic clonic seizure occurred at the age of seven years preceded by an aura of rising abdominal pain and followed by post ictal fatigue. Generalized tonic clonic seizures recurred at weekly interval with and without the preceding abdominal pain. At times the abdominal pain occurs with no generalized tonic clonic seizures but followed by lack of concentration and fatigue. The seizures occurred mainly during wakefulness but could occur during sleep. The patient never achieved seizure remission and never developed status epilepticus despite frequent seizures. He was admitted to the epilepsy monitoring unit EMU at the age of twelve years. He was on two antiepileptic drugs namely topiramate one hundred milligram twice daily and lamotrigine two hundred milligram twice daily with almost weekly seizures. His neurological, general and systemic examinations were normal. The patient was kept on his antiepileptic drugs and had five days video electroencephalography EEG monitoring using ten-twenty system scalp EEG electrodes with additional anterior temporal electrodes $\mathrm{T} 1$ electrode on the left anterior temporal region and T2 electrode on the right anterior temporal region respectively. During the video EEG monitoring the patient developed four stereotyped electroclinical seizures. Three were during wakefulness and one during sleep. No generalized tonic clonic seizures recorded. The seizures were preceded by abdominal pain in one seizure only. The seizure semiology starts with abrupt asymmetric abduction of both upper and lower limbs with prominent tonic and dystonic contraction of the left upper and lower limbs and 
adversive eyes and head deviation to the left with proximal automatic movements of the right arm and leg. The duration of seizure was fifty to sixty seconds during which the patient was unresponsive with early recovery of comprehension and speech in the post ictal phase. The ictal EEG onset starts with right frontal regional suppression followed by slow activity of the right hemisphere in all recorded seizures. The interictal EEG showed asymmetric background with excessive slow transients of three to five hertz activity in the right hemisphere with frequent repetitive interictal spikes and polyspike-wave discharges at right frontopolar region intermixed with two to three slow transients (Figure 1). The electroclinical seizures recorded favored right hemispheric epilepsy with early engagement of the right frontal regions. Preadmission MRI of the brain showed right hippocampal sclerosis HS. Ictal single-photon emission computed tomography SPECT with technetium-99m hexamethyl-propylene amine oxime Tc-99m HMPAO injected at twenty five seconds after ictal EEG onset of the fourth seizure showed right medial and lateral temporal lobe hyperpefusion (Figure 2). Interictal positron emission tomography with F-18 fluorodeoxyglucose F-18 FDG-PET showed right hemisphere hypometabolism with marked reduction of F-18-FDG metabolism at right frontal, right temporal, right parietal and right thalamic regions (Figure 3). As the initial MRI diagnosis of right HS could not alone explain the seizures of the patient with a high probability of additional right hemispheric neocortical focus a follow up high resolution three Tesla MRI brain was performed prior to invasive EEG recording. The MRI brain clearly demonstrated right temporal pole cortical dysplasia in addition to the right HS (Figure 4). The diagnosis of dual pathology was made with right temporal pole cortical dysplasia and right HS the so called HS+. The patient underwent directly epilepsy surgery without invasive EEG implantation .He received extensive right temporal lobectomy under intraoperative electrocorticography and regions with active spiking were removed (Figure 5). Histopathology of the resected right hippocampus showed HS and tissue from the right temporal pole confirmed the presence of focal cortical dysplasia type III. The patient had no post surgical complications. The patient became seizure free and six month post epilepsy surgery interictal EEG was normal. Currently the patient is seizure free with significant cognitive improvement at five years post epilepsy surgery on lamotrigine $25 \mathrm{mg}$ once daily .He attends high school and plans to be a teacher.

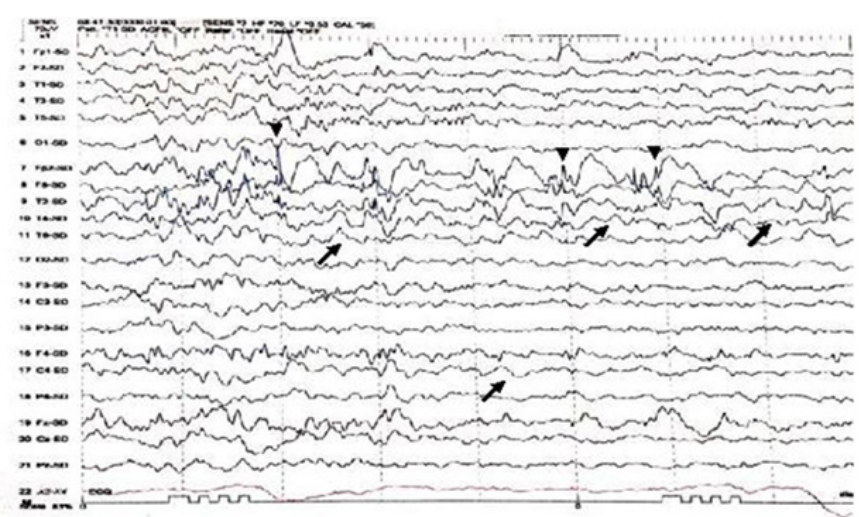

Figure I Interictal EEG shows asymmetry of EEG background with excessive slow transients of 2 to $3 \mathrm{Hertz} \mathrm{Hz}$ in the right hemisphere (black arrows). Intermixed spikes and poly spikes wave discharges at the right fronto-polar regions FP2 (arrow heads).
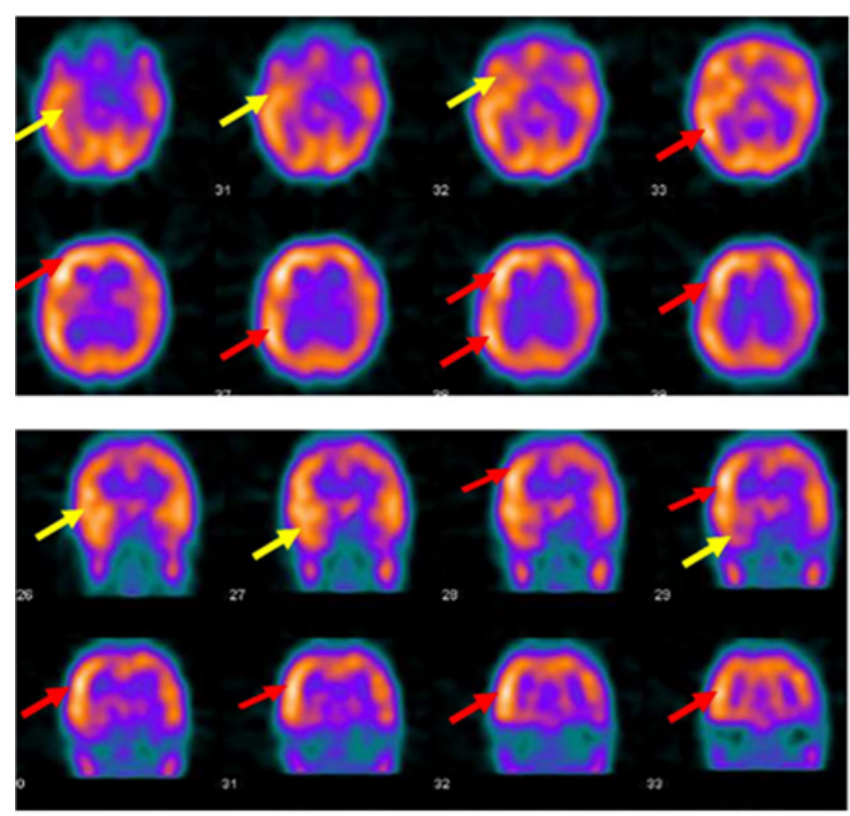

Figure 2 Ictal SPECT with Tc-99m HMPAO injected at twenty five seconds after ictal EEG onset of the fourth seizure shows right medial and lateral temporal lobe hyperpefusion (yellow arrows) and right frontal and right parietal hyperperfusion ( red arrows).
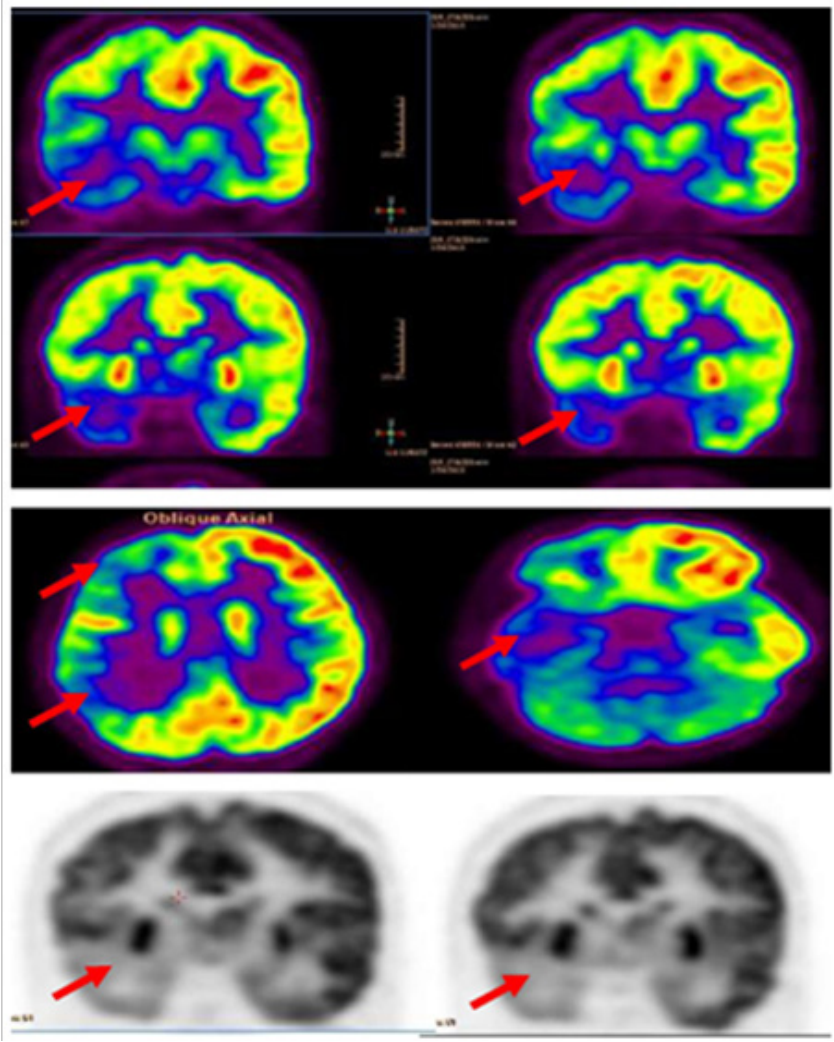

Figure 3 Interictal F-18 FDG-PET showed right hemisphere hypometabolism with marked reduction of F-18-FDG metabolism at right frontal, right temporal, right parietal and to less degree right thalamic regions (red arrows). 


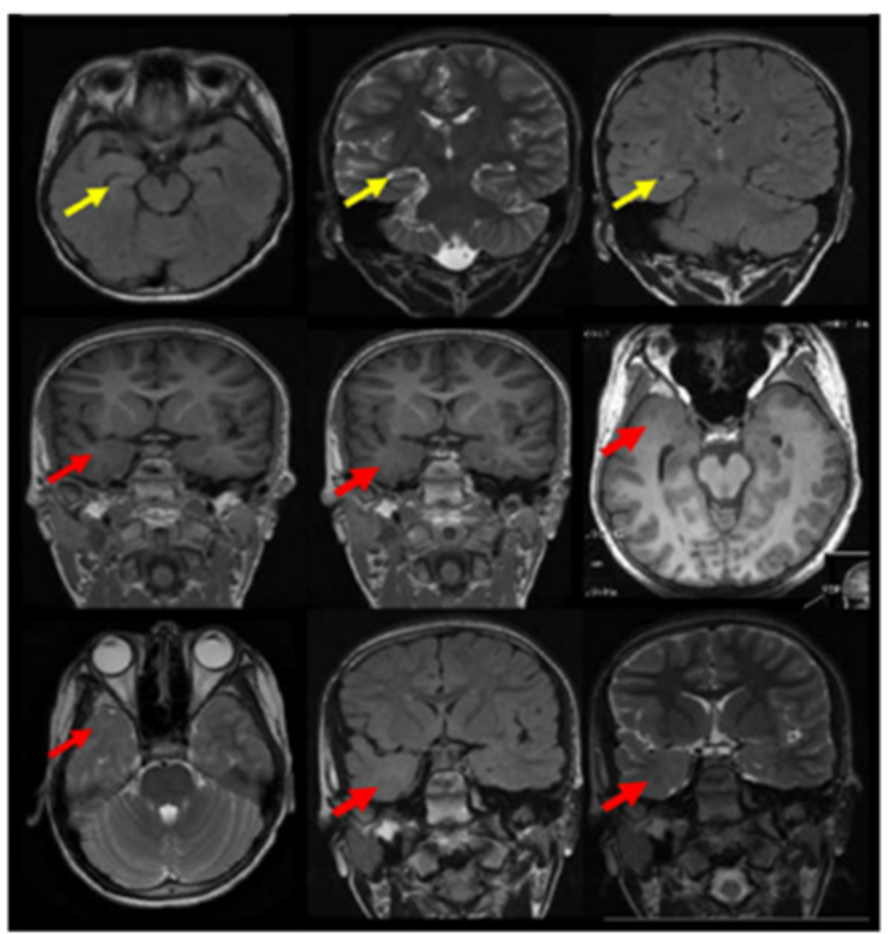

Figure 4 The MRI brain demonstrates right temporal dual pathology. Right HS with atrophic changes of the right hippocampus with architecture distortion and increased signal intensity on coronal T2 and FLAIR and axial FLAIR weighted images is demonstrated ( yellow arrows).In addition right temporal pole cortical dysplasia is shown with smaller right temporal pole ,blurred grey white junction in the right temporal pole on axial and coronal three-dimensional spoiled gradient recalled acquisition in steady state 3-DSPGR images and smaller right temporal pole with blurred grey white matter junction and increased signals on axial T2 and coronal T2 and FLAIR weighted images (red arrows).
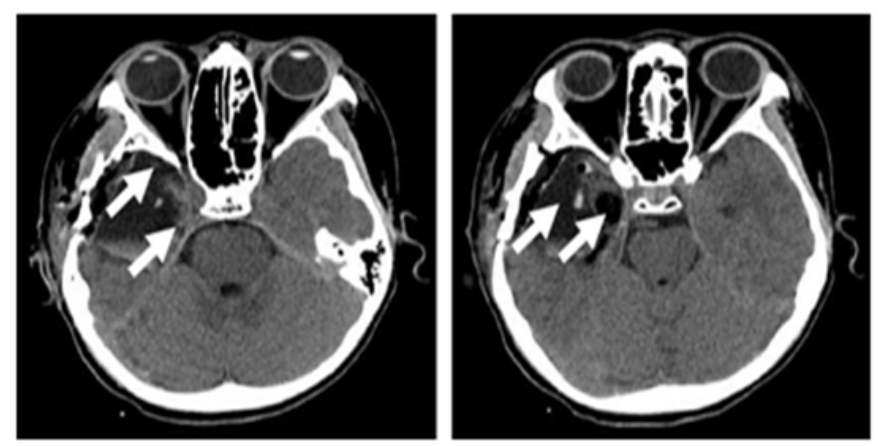

Figure $\mathbf{5}$ The CT scan brain one day after epilepsy surgery demonstrates the extent of the surgical resection with removal of the HS and the temporal pole FCD (white arrows).

\section{Discussion}

Clinical and research studies suggest the important and underappreciated role of the temporal pole in the genesis and propagation of seizures in temporal lobe epilepsy TLE. Electrocorticographic recordings of the TP in patients with pharmacoresistant TLE demonstrate the crucial role of the TP cortex in temporal lobe seizure networks. ${ }^{1}$ The association of HS and macroscopic or microscopic focal cortical dysplasia in the temporal neocortex TN is often reported in pharmaco-resistant temporal lobe epilepsy. ${ }^{2}$
This combination of dual pathology contributes to epileptogenesis with a role of both pathologies in the generation of ictal and interictal activity. ${ }^{2}$ Simultaneous invasive EEG recordings from the hippocampus and the TN support the epileptogenicity of the dysplastic tissue in the TN. ${ }^{2}$ The degree of HS quantify the degree of contribution of the hippocampus to seizure generation whereas the subtype of the FCD and the location within the TN does not influence the degree of epileptogenicity of the FCD or its role in seizure generation. $^{2}$ Mesial temporal lobe epilepsy with HS is considered as a clinical syndrome with distinct clinical, neurophysiological and neuroimaging features. ${ }^{3}$ Therefore the presence of peculiar clinical or neurophysiological features in the phase one presurgical evaluation data of patients with HS on the MRI and the patient presented in this case report is an illustrative example warrants further investigations to rule out dual pathology with additional epileptogenic lesions affecting the extrahippocampal or extratemporal regions. ${ }^{3} \mathrm{HS}$ association with FCD that is, HS+ is common particularly in children and temporal polar TP cortex is frequently involved in seizure onset. ${ }^{2,3}$ Patients with dual pathology may have favorable outcome after epilepsy surgery provided that both pathologies are removed. ${ }^{3}$ The three-tiered ILAE classification system of FCD distinguishes isolated forms (FCD types I and II) from those associated with another principal lesion, that is, HS and FCD type IIIa, tumors and FCD type IIIb, vascular malformations and FCD type IIIc, or lesions acquired during early life (i.e., traumatic injury, ischemic injury, or encephalitis and FCD Type IIId. ${ }^{4}$ MRI of the brain has proven its precision as a diagnostic tool for recognition of different structural lesions underlying medically intractable seizures. ${ }^{5}$ There is strong evidence that preoperative MRIidentified hippocampal atrophy consistent with HS concordant with the seizure origin in the temporal lobe is a significant factor associated with a favorable outcome. PET studies may be valuable in individuals with unremarkable MRI findings. ${ }^{5}$ For accurate evaluation MRI brain imaging epilepsy protocol should be used in 1.5 or 3 Tesla MRI scanner. ${ }^{4}$ The patient is placed in a supine position with the head immobilized within the head coil. Twenty-four coronal slices are acquired using a T2 turbo spin echo TSE sequence $(\mathrm{FOV}=160 \mathrm{~mm}, \mathrm{TE}=92 \mathrm{msec}$, $\mathrm{TR}=4250 \mathrm{msec}$, slice thickness $3.0 \mathrm{~mm}$, voxel size $0.6 \times 0.5 \times 3.0 \mathrm{~mm}^{3}$ ) perpendicular to the hippocampi, from the genu to the splenium of the corpus callosum; also coronal T2 fluid-attenuated inversion recovery (FOV $=230 \mathrm{~mm}, \mathrm{TE}=86 \mathrm{msec}, \mathrm{TR}=9000 \mathrm{msec}$, slice thickness $5 \mathrm{~mm}$, voxel size $1.1 \times 0.9 \times 5.0 \mathrm{~mm}^{3}$ ) and $\mathrm{T} 2$ short tau inversion recovery (FOV $=180 \mathrm{~mm}, \mathrm{TE}=71 \mathrm{msec}, \mathrm{TR}=3070 \mathrm{msec}$, slice thickness $5 \mathrm{~mm}$, voxel size $0.8 \times 0.7 \times 5.0 \mathrm{~mm}^{3}$ ) sequences are acquired, planned over an axial plane, perpendicular to the temporal lobe, from the anterior horn to the posterior horn of the lateral ventricles. ${ }^{5}$ FCD can be easily missed with suboptimal MRI protocols especially when the FCD is in the temporal pole or the FCD is subtle. ${ }^{6}$ To improve MRI brain visualization of temporal pole pathology including subtle FCD the MRI technique should include three-dimensional (3D) fluid-attenuated inversion recovery FLAIR/T1-weighted images, coronal $3 \mathrm{~mm}$ slices with T2-weighted and FLAIR sequences, and $2 \mathrm{~mm}$ axial T2-weighted images. ${ }^{7}$ Common features of FCD on MRI include: increased focal cortical thickening or thinning, focal brain atrophy, blurred graywhite junction, increased signal on T2 weighted and FLAIR-weighted images in the gray matter of the brain including the temporal pole and at times embedding of gray matter lesions in the subcortical white matter. ${ }^{6}$ Published studies showed temporo-polar MRI abnormalities are detected in one- to two-thirds of all patients suffering from TLE.? Focal cortical dysplasia Type IIIa FCD IIIa, a developmental lesion resulting from defects in neuronal formation and migration into 
the temporal pole FCD I is often seen with HS.,7 Other types of pathology in the temporal pole such as post-inflammatory changes and small temporal pole encephalocele also described in the temporal pole with HS. ${ }^{8}$ Patients with histopathological proven FCD IIIa had a significantly better seizure outcome after anterior mesial temporal lobe resection AMTLR than patients with HS alone. ${ }^{9}$ MRI brain is very helpful to identify patients with pharmaco-resistant temporal lobe epilepsy who are likely to benefit from epilepsy surgery and can spare the patient invasive EEG recording when identifies surgically remediable lesions similar to our patient with dual pathology. ${ }^{10}$ Therefore its important to exclude temporal pole abnormalities when evaluating MRI brain in TLE. The temporal pole MRI abnormalities include blurring of the gray-white matter junction with regionally decreased myelination and white matter hyperintensity. The blurring involves the whole temporal pole, not just the area of FCD ${ }^{11}$ This can help identifying the extent of epileptogenic foci in TLE and patients with TLE due to FCD are eighteen times more likely to have more pronounced findings on MRI than patients with extratemporal epilepsy. ${ }^{11}$ With the progressive refinement of MR techniques FCDs are being seen in vivo with increasing frequency in patients with epilepsy and many previously considered non lesional epilepsies are now recognized with associated FCD or other malformations of cortical development MCD. Currently there is a growing interest and development in the MRI characterization of FCD and other MCD to assist electroclinical localization and surgical planning with less invasive procedures. ${ }^{10,1}$

\section{Supplementary materials}

Power Point file with the figures of the case report and their legands The file has 5 figures that include inter ictal EEG, brain ictal SPECT scan, brain interictal PET scan, preoperative brain MRI and post operative brain CT scan.

\section{Acknowledgements}

None.

\section{Conflict of interest}

The author declares that there is no conflict of interest regarding the publication of this paper.

\section{References}

1. Abel TJ, Woodroffe R, Moritani T. The Role of the Temporal Pole in Temporal Lobe Seizure Networks: An Intracranial Electrode Investigation. Neurosurgery. 2016;63(Suppl 1):194.

2. Fauser S, Schulze-Bonhage A. Epileptogenicity of cortical dysplasia in temporal lobe dual pathology: an electrophysiological study with invasive recordings. Brain. 2006;129(Pt 1):82-95.

3. Marusic P, Tomasek M, Krsek P, et al. Clinical characteristics in patients with hippocampal sclerosis with or without cortical dysplasia. Epileptic Disord. 2007;9 (Suppl 1):S75-82.

4. Blumcke I, Thom M, Aronica E, et al. The clinicopathologic spectrum of focal cortical dysplasias: a consensus classification proposed by an ad hoc Task Force of the ILAE diagnostic methods commission. Epilepsia. 2011;52(1):158-174.

5. Jones AL, Cascino GD. Evidence on use of neuroimaging for surgical treatment of temporal lobe epilepsy: a systematic review. JAMA Neurol. 2016;73(4):464-470.

6. Guidelines for neuroimaging evaluation of patients with uncontrolled epilepsy considered for surgery. Commission on Neuroimaging of the International League Against Epilepsy. Epilepsia. 1998;39(12):13751376.

7. Ryvlin P, Coste S, Hermier M, et al. Temporal pole MRI abnormalities in temporal lobe epilepsy. Epileptic disord. 2002;4(Suppl 1):33-40.

8. Toledano R, Jim A, Huete E, et al. Small temporal pole encephalocele: A hidden cause of "normal" MRI temporal lobe epilepsy. Epilepsia. 2016;57(5):841-851.

9. Dührsen L, Sauvigny T, House PM, et al. Impact of focal cortical dysplasia Type IIIa on seizure outcome following anterior mesial temporal lobe resection for the treatment of epilepsy. J Neurosurg. 128(6):1668-1673.

10. Kabat J, Krol P. Focal cortical dysplasia-review. Pol $J$ Radiol. 2012;77(2):35-43.

11. Bartolini L, Whitehead MT, Ho CY, et al. Temporal lobe epilepsy and focal cortical dysplasia in children: A tip to find the abnormality. Epilepsia. 2017;58(1):113-122. 